\title{
O desenvolvimento da roupa biocinética
}

\author{
Ana Irene Alves de Oliveira ${ }^{a}$, Larissa Santos Prazeres ${ }^{b}$ \\ ${ }^{a}$ Terapeuta ocupacional, doutora em Teoria \& Pesquisa do Comportamento, docente do curso de Terapia \\ Ocupacional, Universidade do Estado do Pará - UEPA, coordenadora do Núcleo de Desenvolvimento em \\ Tecnologia Assistiva e Acessibilidade - NEDETA, Belém, PA, Brasil \\ ${ }^{\mathrm{b}}$ Acadêmica do $5^{\circ}$ ano do curso de graduação em Terapia Ocupacional, Universidade do Estado do Pará - UEPA, \\ estagiária do Núcleo de Desenvolvimento em Tecnologia Assistiva e Acessibilidade - NEDETA, Belém, PA, Brasil
}

\begin{abstract}
Resumo: A ideia de promover o desenvolvimento de dispositivos de tecnologia assistiva economicamente acessíveis preconizada no trabalho proposto pelo Núcleo de Desenvolvimento em Tecnologia Assistiva e Acessibilidade - NEDETA permitiu conhecer a importância, os benefícios e as dificuldades da utilização de trajes na reabilitação de crianças com deficiência neuromotora e motivou o desenvolvimento deste estudo. Buscou-se desenvolver um dispositivo que se aproximasse da realidade climática da região Norte e utilizasse materiais economicamente acessíveis, auxiliando na (re)habilitação de crianças com deficiências neuromotoras. O trabalho configura-se como uma pesquisa do tipo aplicada de desenvolvimento tecnológico e inovação (P\&DI), uma vez que propõe um estudo experimental do desenvolvimento de uma órtese dinâmica (roupa biocinética) a partir das características neuromotoras de duas crianças de uma instituição de acolhimento para crianças com deficiência, durante o período de maio à novembro de 2012. Foram desenvolvidos cinco protótipos da roupa biocinética que, por sua vez, foram observados e modificados durante o período da experimentação. Percebeu-se que tais protótipos possibilitaram maior suporte corporal, o favorecimento da estabilidade e, ainda, proporcionaram input de estímulos proprioceptivos e a inibição de padrões patológicos pertinentes à deficiência neuromotora. Durante a experimentação, foi possível reforçar a importância da utilização de órteses dinâmicas, enquanto roupas, no tratamento de crianças com deficiência neuromotora, logo, ratificando a relevância para a proposta de confecção da roupa biocinética.
\end{abstract}

Palavras-chave: Paralisia Cerebral, Equipamentos de Autoajuda, Tecnologia Assistiva, Reabilitação.

\section{The development of biokinetics clothing}

\begin{abstract}
Introduction: The idea of promoting the development of affordable assistive technology devices advocated in the work proposed by the 'Núcleo de Desenvolvimento em Tecnologia Assistiva e Acessibilidade' (NEDETA) alerted for the importance, benefits, and difficulties of using costumes in the rehabilitation of children with neuromotor disabilities, and motivated the development of this study. Objective: Given this, we sought to develop a device that would fit the reality of the North region climate and used affordable materials to assist the rehabilitation of children with neuromotor disabilities. Method: To this end, the research was configured as P\&DI type, because it proposed an experimental study to develop a dynamic device (biokinetics clothing) based on the neuromotor characteristics of two children from a host institution for the disabled, between May and November 2012. Results: Thus, five prototypes were developed, biokinetics clothing. The prototypes were observed and modified during the experiment. It was possible to realize that such prototypes allowed greater body support, favored stability, provided input to proprioceptive stimulation, and inhibited the pathological patterns relevant to neuromotor disabilities. Conclusion: During the trial, it was possible to reinforce the importance of using dynamic orthoses as clothes to treat children with neuromotor disabilities, thus confirming the relevance of the proposal to manufacture biokinetics clothing.
\end{abstract}

Keywords: Cerebral Palsy, Self-help Devices, Assistive Technology, Rehabilitation.

Autor para correspondência: Ana Irene Alves de Oliveira, Núcleo de Desenvolvimento em Tecnologia Assistiva e Acessibilidade - NEDETA Universidade do Estado do Pará, Trav. Perebebuí, s/n, sala 12, Campus II, Bloco D/UEAFTO, Marco, CEP 66087-670, Belém, PA, Brasil, e-mail: anairene25@gmail.com

Recebido em 4/12/2012; Aceito em 18/1/2013. 


\section{Introdução}

A paralisia cerebral (PC) é uma desordem de base sensório-motora que envolve os distúrbios do tônus muscular, postura e movimentos voluntários, ocasionada por lesão cerebral, comumente associada com hipóxia e/ou anóxia no período de maturação estrutural e funcional do cérebro. Em relação à incidência, estimam-se índices de 1,5 a 2,5 para cada mil nascidos vivos nos países desenvolvidos, e de 7 para cada mil nascidos vivos em países em desenvolvimento. No Brasil, os dados apontam cerca de $30 \mathrm{mil}$ a $40 \mathrm{mil}$ casos novos por ano (MANCINI et al., 2002).

Por sua vez, a mielomeningocele (MMC) é uma malformação que ocorre até a quarta semana de embriogênese, responsável por cerca de $85 \%$ dos distúrbios do fechamento do tubo neural (DFTN) presentes ao nascimento, caracterizando-se por falha no fechamento dos arcos vertebrais posteriores, levando à exposição do tecido nervoso, que resulta em crescimento displásico de estruturas do sistema nervoso central, formando uma bolsa cística ao nível da lesão (MOURA et al., 2011).

As deficiências neuromotoras frequentemente culminam em um atraso no desenvolvimento neuropsicomotor, em virtude de uma lesão no sistema nervoso central, que provoca comprometimentos na área motora, sensorial e/ou cognitiva, implicando em alteraçóes de tônus muscular, qualidade de movimento, percepçóes e capacidade de apreender e interpretar os estímulos ambientais (ALVES DE OLIVEIRA, 2008).

O quadro clínico mais frequente traz alteraçôes tônicas e padróes anormais de coordenação muscular. Ubaldo, Medeiros e Brito (2002) citam o aumento do tônus muscular como fator responsável por levar o paciente a adotar posturas anormais com movimentos restritos, contração inadequada, limitação funcional em adotar determinadas posturas e propensão às instalaçóes de padróes posturais patológicos, criando assim um desequilíbrio muscular.

Frente às possíveis sequelas no plano sensoriomotor da $\mathrm{PC}$ e da MMC, diversos métodos são empregados no tratamento, o conceito neuroevolutivo Bobath, a facilitação neuromuscular proprioceptiva, integraçáo sensorial, dentre outros. Contudo, além dos métodos utilizados em terapias, faz-se necessária a existência de dispositivos de manutenção dos ganhos e de prevenção de deformidades, nos outros contextos da criança com deficiência neuromotora. Dessa forma, os dispositivos de tecnologia assistiva têm sido amplamente utilizados a fim de se favorecer autonomia e independência para os indivíduos com dificuldades motoras.

Estudos têm mostrado a eficácia dos trajes como o Theratogs (2012), SPIO (2012), além de métodos como o TheraSuit e PediaSuit, utilizados como órteses $^{1}$ na assistência a crianças com deficiência neuromotora. O TheraSuit, por exemplo, foi estudado por Koscielny (2004) como recurso terapêutico no tratamento de crianças com paralisia cerebral. Em sua pesquisa, verificou-se que os principais objetivos da vestimenta são a normalização do tônus muscular da criança, aumentar a variedade dos movimentos ativos, aumentar a força e a resistência e controlar os grupos musculares que acabaram de ganhar força, permitindo que a criança melhore suas habilidades (THERASUIT..., 2002).

A partir das pesquisas supracitadas, pôde-se perceber a necessidade de se desenvolver outra variedade de dispositivo do tipo órtese dinâmica que auxiliasse a (re)habilitação de crianças com deficiência neuromotora e, ainda, considerasse não apenas as características pertinentes a essa deficiência, e sim as características do contexto onde cada possível usuário está inserido. Assim, o estudo em questáo tem por objetivo apresentar o desenvolvimento dos protótipos do traje denominado roupa biocinética, que auxilia no tratamento de sujeitos pertencentes a uma instituição especial de acolhimento a crianças com deficiência. Ainda, que o equipamento atenda, além das necessidades biomecânicas e neuromotoras, os aspectos climáticos da região Norte a partir do uso de tecidos alternativos e economicamente acessíveis, logo, justificando e mostrando à comunidade acadêmica a importância do uso desse dispositivo como recurso de tecnologia terapêutica para a melhoria do desempenho funcional dessa demanda.

\section{Procedimentos metodológicos}

Este estudo é classificado, de acordo com sua natureza, como pesquisa aplicada ou tecnológica, pois, segundo Silva e Zambalde (2008, p. 39), "[...] é o tipo de estudo que objetiva a aplicação dos conhecimentos básicos na geração de novos produtos, processos, patentes e serviços". Quanto a seus objetivos, a pesquisa é classificada como exploratória, pois "[...] possibilita ao pesquisador aumentar sua experiência em torno de determinado problema [...]" (PINHEIRO, 2010, p. 27). Ainda segundo o autor, ela abrange levantamento bibliográfico; entrevista com pessoas que tiveram experiências práticas com o problema pesquisado; e análise de exemplos que estimulam a compreensão. 
O estudo foi iniciado com atividades de pesquisa e levantamento bibliográfico, análise e observaçáo, sobre os tipos de órteses dinâmicas, especificamente os trajes já utilizados na reabilitação de crianças com deficiência neuromotora. A partir do referencial teórico da pesquisa, obtido por meio de livros, revistas, monografias, periódicos, entre outros documentos, foi ampliado o conhecimento relacionado à carência do uso de tais dispositivos na regiấo Norte. A proposta da roupa biocinética foi desenvolvida mediante estudo da situação das crianças com deficiência neuromotora de um abrigo da região, seguindo o fluxograma descrito na Figura 1.

O presente estudo reporta uma metodologia de gestáo de projetos de pesquisa, desenvolvimento \& inovação, baseada no direcionamento dos projetos de pesquisa para a obtenção de produtos.

\section{Resultados}

Através da revisão de literatura, percebeu-se que os trajes utilizados na reabilitação de crianças com deficiências neuromotoras ainda não fazem parte do cotidiano dessa população. A proposta de confecção da roupa biocinética surgiu depois da pesquisa de outras semelhantes existentes no mercado.

Observou-se que além de apresentar um custo muito elevado, por ser tecnologia importada, os tecidos das roupas já comercializadas eram bastante desconfortáveis para as condiçôes climáticas da regiâo Norte. O nome (biocinética) é inspirado nas características biomecânicas, somado ao movimento, pois mesmo vestida a criança tem a liberdade de movimentar-se, visto que é uma órtese dinâmica.

Foram construídos, até o fechamento deste, cinco protótipos da roupa biocinética, de diferentes modelos, tecidos, posicionamento das presilhas e tiras elásticas. Os protótipos foram desenvolvimentos considerando as características neuromotoras de duas crianças do abrigo (Figura 2). As roupas foram experimentadas e ajustadas. A fase de teste da eficácia da roupa ainda será iniciada. Os protótipos foram evoluindo na medida em que se observaram seus pontos positivos ou negativos durante a experimentação.

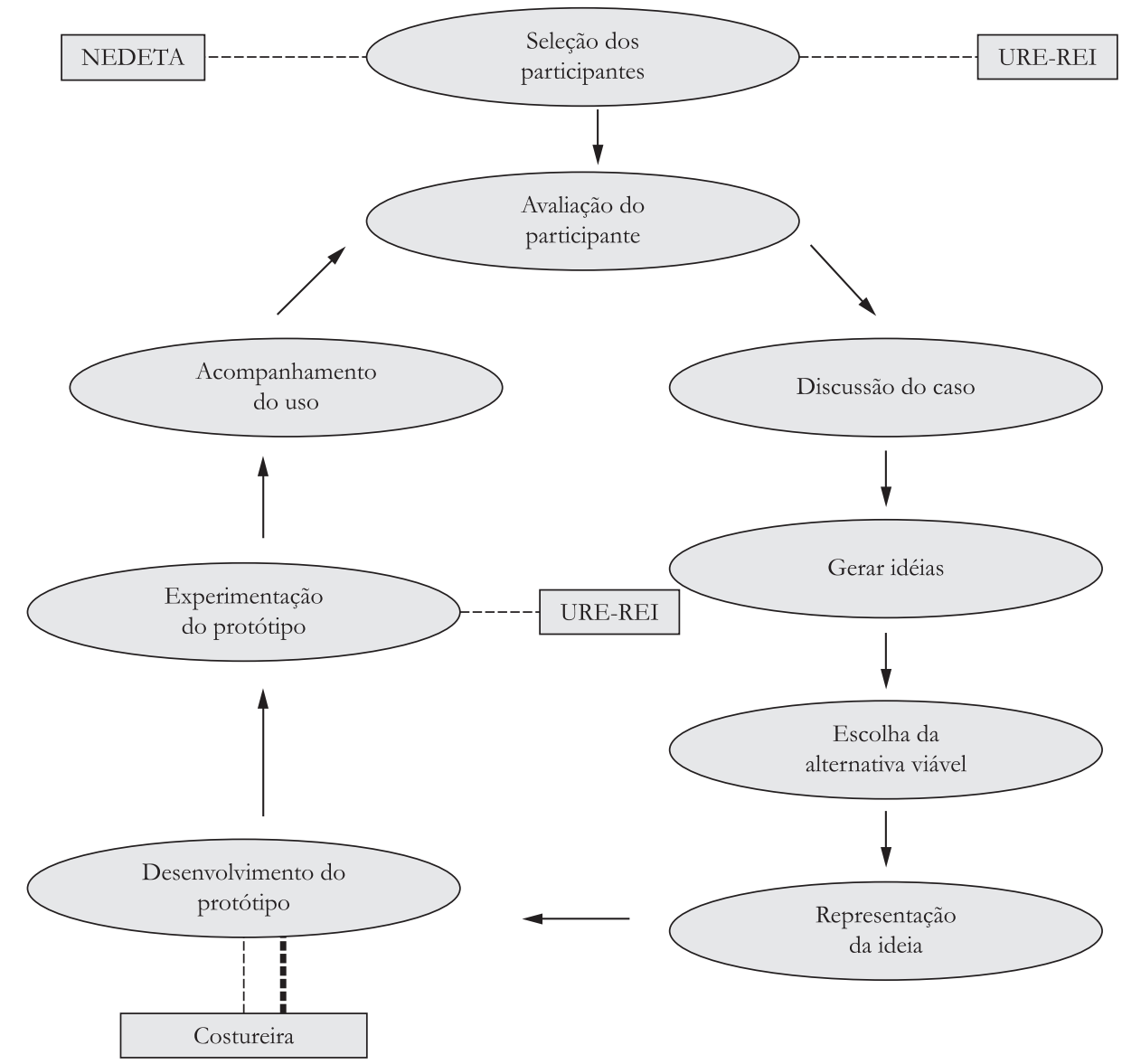

Figura 1. Fluxograma de gestão do produto. 
A Figura 2a mostra os croquis iniciais da roupa, pensada como um modelo comum do vestuário infantil conhecido como macacão. Após a finalização do desenho inicial, deu-se início à pesquisa de tecidos, presilhas e elásticos, materiais que seriam utilizados. O primeiro tecido escolhido foi o jeans, a exemplo do TheraSuit, um tipo de roupa confeccionada em jeans, e posteriormente o neoprene. Contudo, tais opções logo foram descartadas considerando o desconforto que causariam no usuário.

O primeiro tecido utilizado na confecção da roupa biocinética foi helanca ${ }^{2}$, mas foi descartado, pois não oferecia o suporte corporal necessário para os resultados esperados. O primeiro protótipo (Figura 2b) apresentava um modelo diferente do inicial, pensado primeiramente para crianças sem comprometimentos em membros superiores, ainda sem as presilhas fixadas e os elásticos para realizar as traçôes. Esse primeiro modelo também logo foi descartado, pois acreditava-se que ao realizar a tração o modelo ficaria desconfortável no usuário.

Ainda utilizando a helanca, foi confeccionado o segundo protótipo (Figura 2c), agregando presilhas de metal no formato retangular e utilizando elástico para realizar a tração. Observou-se que, ao ser tracionado,
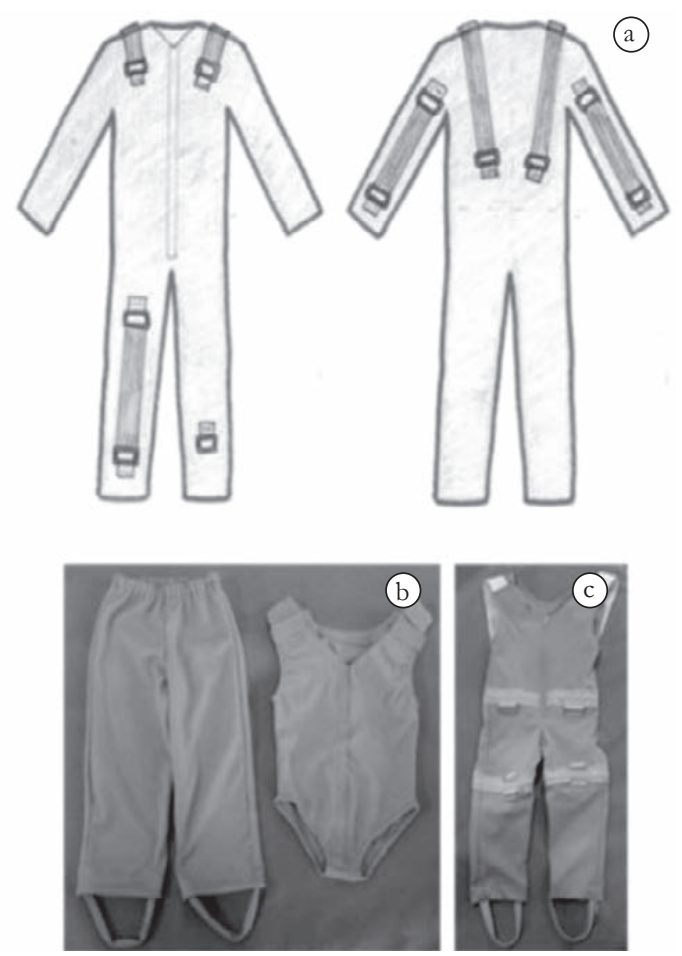

Figura 2. a) Croqui do projeto; b) Protótipo número 1; c) Segundo protótipo agregando presilhas de metal no formato retangular e utilizando elástico para realizar a tração. o tecido da roupa ficava demasiadamente alongado, dessa forma nâo seria possível estabilizar o tronco.

Ao se descartar os protótipos que utilizaram helanca, optou-se por outro tecido: a Lycra ${ }^{\circledR 3}$ compressiva. Tal tecido tem sido utilizado na confecção de cintas, necessárias após algum procedimento cirúrgico. O terceiro protótipo (Figura 3a) já apresenta o novo tecido, novos formatos de presilha, pois o anterior foi descartado por apresentar uma abertura, o que poderia deformá-lo ao longo do tempo de uso. Os elásticos também foram reforçados, sendo cobertos com o próprio tecido da roupa.

Observou-se, dessa forma, que o novo tecido oferecia melhor suporte corporal e, consequentemente, melhor estímulo proprioceptivo, baseado nos princípios do conceito Bobath, FNP e IS. O controle postural é uma habilidade complexa, que requer a integração de informaçóes aferentes, com o sistema motor presente nos diversos níveis do sistema nervoso, somados a um sistema musculoesquelético íntegro para realizar os comandos.

As mudanças do protótipo 2 para o 3 começaram a apresentar bom resultado, sendo selecionada, então, a Lycra compressiva como tecido da roupa biocinética. Após a seleção do tecido, fez-se necessário realizar ajustes no posicionamento das presilhas, para que se chegasse ao melhor alinhamento corporal, e sentiu-se a necessidade da confecção com dupla face para que proporcionasse mais compressão. Dessa forma, o protótipo 4 (Figura $3 \mathrm{~b}$ ) traz algumas mudanças na colocação das presilhas para alinhamento do tronco, quadril e extensão de membros inferiores.

Em relação ao tronco, as presilhas foram fixadas de modo que as tiras elásticas pudessem respeitar as
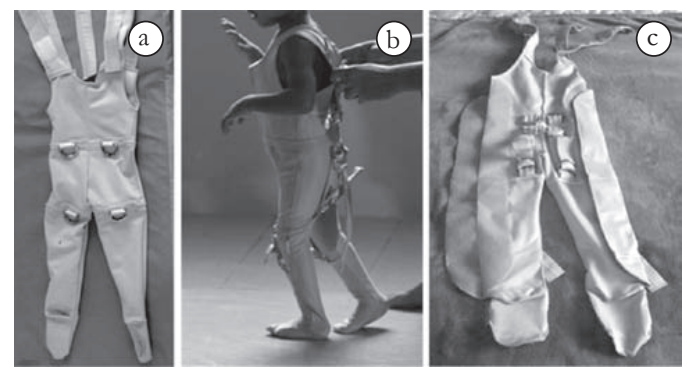

Figura 3. a) Protótipo $3 \mathrm{com}$ as presilhas da parte superior fixadas mais distante da linha média; b) Protótipo 4 com posição das presilhas corrigida, de modo a respeitar a direção das fibras do glúteo máximo; c) Protótipo 5 com inserção de velcro nas laterais, possibilitando a abertura em ambos os lados; as presilhas e tiras elásticas não foram alteradas. 
cadeias musculares responsáveis pelo alinhamento do tronco. As tiras elásticas foram fixadas anteriormente pouco abaixo da clavícula, seguindo para a parte posterior, sendo cruzadas posteriormente, respeitando a direção das fibras do músculo trapézio.

Outro ajuste realizado do protótipo 3 para o protótipo 4 foi nos pontos onde são fixadas as presilhas para corrigir o quadril. No protótipo 3 as presilhas da parte superior eram fixadas mais distante da linha média. Observou-se que dessa forma, quando vestida a roupa, as tiras elásticas saíam da posição mais adequada, não realizando a extensão do quadril. No protótipo 4, a posição das presilhas foi corrigida, de modo que foi possível respeitar a direção das fibras do glúteo máximo alcançando o objetivo.

O terceiro ajuste do protótipo 3 para o 4 foi a mudança das posições das presilhas para promover a extensão do joelho. Inicialmente, as presilhas foram fixadas acima do joelho e anteriormente na direção da articulação da tíbia com o tálus. No protótipo 4, a posiçáo superior das presilhas foi mantida, contudo o outro ponto de fixaçáo foi logo abaixo da articulaçáo do joelho, alcançando o resultado.

O protótipo 5 apresentou mudanças no modelo da roupa, pois foram inseridos velcros nas laterais, possibilitando a abertura em ambos os lados. As presilhas e tiras elásticas não foram alteradas. Observou-se que nessa nova mudança houve maior facilidade na colocação da roupa, além de permitir que ela se ajuste a mais de uma criança. As laterais do protótipo em questáo passaram ser abertas e fechadas através de velcro.

\section{Discussão}

A roupa biocinética tem por objetivo favorecer melhor alinhamento e estabilidade biomecânica. Ao se experimentar a roupa, observou-se que tal objetivo foi alcançado. As crianças que serviram de modelo participam de atendimentos fisioterápicos e terapêuticos ocupacionais, entre outros. Contudo, verifica-se a necessidade de um dispositivo que possibilite a manutenção dos ganhos obtidos com as terapias e suporte corporal em outros momentos, na perspectiva da plasticidade cerebral.

As crianças com deficiência neuromotora apresentam uma alteração significativa no alinhamento biomecânico durante a manutenção de posturas estáticas e dinâmicas, implicando em uma sobrecarga no sistema musculoesquelético e que se agrava com o crescimento da mesma, acentuando o risco para o aparecimento de encurtamentos musculares que podem se tornar futuras deformidades articulares ou ósseas (ASSUMPÇÃO, 2005).

Sabe-se que os padróes de tônus muscular e de movimento influenciam diretamente no controle postural; agregado a isso, os sistemas vestibular, visual e proprioceptivo têm um papel fundamental, pois conduzem informaçóes ao sistema nervoso que são integradas em vários níveis e comparadas com a memória, permitindo que muitas áreas do córtex cerebral participem do desenvolvimento da resposta, a qual é transmitida ao sistema musculoesquelético para a produção de movimentos dos olhos, cabeça, tronco e membros, tendo por objetivo a geraçáo de movimentos coordenados das posturas estáticas e dinâmicas (KONRAD; GIRARDI; HELFERT, 1999).

Verificou-se, dessa forma, que a escolha do tecido foi fundamental para o alcance dos objetivos da roupa. $\mathrm{O}$ tecido selecionado oportunizou para os usuários da roupa suporte e estímulo tátil, o que se pode perceber no trabalho de Adler, Beckers e Buck (2007), que sugerem que uma pressão aplicada no músculo melhora a sua capacidade de contração; assim, proporcionar uma pressão em oposição à direção do movimento estimula a musculatura sinérgica, reforçando a contração. Os estímulos táteis aplicados no tronco do paciente facilitam o movimento dos músculos e dos membros por meio da promoção da estabilidade do tronco.

Rodrigues, Cavalcanti e Galvão (2007) sugerem que no processo de seleção do material é necessária a análise de sua aplicação para que entấo se possa determinar a característica mais importante que ele deve possuir. Tal análise passa desde a condiçáa neuromotora do indivíduo até os resultados que se almeja. Os terapeutas devem estar seguros das condições do material, se este é resistente, frágil ou dúctil, além de analisar se o material estará sujeito à aplicação de grande força, altos níveis de tensão, temperaturas.

Em relação ao tronco, o objetivo é corrigir padrões de escoliose e cifoses. Kendall et al. (2007) relata que a coluna vertebral normal apresenta curvas na direçâo anterior e posterior, contudo uma curva posterior anormal caracteriza a cifose, e uma curva na direçáo lateral representa uma escoliose. Pode-se citar algumas causas para a escoliose, podendo ser congênita, resultar de doença ou lesão, podendo relacionar-se a problemas neuromusculares que influenciam diretamente a musculatura do tronco.

As tiras posicionadas para correção de tronco obedecem o sentido do músculo trapézio. Baseado nos conhecimentos do método das cadeias musculares, 
sabe-se que os músculos trabalham em conjunto, assim foi possível alcançar o objetivo de prevenir escolioses e cifoses.

Além das alteraçôes relacionadas ao tronco, Cargnin e Mazzitelli (2003) apontam que as deformidades mais comuns do quadril na paralisia cerebral espástica, por exemplo, são adução, flexão e rotação medial, isoladas ou combinadas entre si. A flexão do quadril pode estar associada à flexão dos joelhos, gerando a posição agachada. A deformidade em flexão-adução surge inicialmente como resultado de um desequilíbrio entre agonistas e antagonistas, podendo agravar-se progressivamente.

Dessa forma, com os devidos ajustes, as tiras elásticas passaram a agir de acordo com as fibras do músculo glúteo máximo, assim, a partir do protótipo 4 , conquistou-se um posicionamento melhor de quadril, auxiliando na retificação de tronco e membros inferiores.

Foi possível observar, ao se experimentar a roupa biocinética, que o dispositivo alcançou os seus objetivos, por estar de acordo com os princípios de inibição de padrôes patológicos, facilitaçáo de movimentos coordenados e estimulação dos métodos de tratamento segundo Bobath, Kabat, cadeias musculares e integração sensorial.

Observou-se ainda com este estudo a importância da utilização dos dispositivos de tecnologia assistiva, especificamente as órteses, na manutenção das habilidades, além da prevenção, do aumento da função das crianças com deficiência neuromotora.

Estudos apontam que o uso de órtese como adjuvante na reabilitação de crianças com paralisia cerebral, por exemplo, tem aumentado significativamente, pelo fato de essa clientela estar sujeita ao desenvolvimento de deformidades acarretando limitações funcionais, por apresentarem padróes patológicos e movimentos articulares anormais devido à presença da espasticidade e do controle motor anormal (ASSUMPÇÃO, 2005).

Através da observação, ao se experimentar a roupa, percebe-se os benefícios do dispositivo. Contudo, como todo processo de prescriçáo de um dispositivo de tecnologia assistiva, ainda se faz necessário acompanhar o uso do protótipo e reavaliar o usuário, a fim de se ter dados para se subsidiar o tempo de utilização da órtese em questão.

\section{Considerações finais}

As deficiências neuromotoras implicam em alteraçôes posturais e de coordenação de movimento, que por sua vez interferem na qualidade de vida, além de provocar alteraçóes na biomecânica corporal. Os encurtamentos e as deformidades articulares surgem por mau posicionamento dos segmentos afetados e pelo desenvolvimento de espasticidade e causam danos na postura e marcha.

As alterações no sistema motor repercutem em alteraçôes do esquema corporal, sendo a soma de todos os eventos que ocorrem nas funções sensitivo-motoras, afetivas e intelectuais e que levam à consciência do próprio corpo. Dessa forma, surge a importância de se utilizar as técnicas consciência e esquema corporal, além de dispositivos como as órteses, para melhorar o desequilíbrio muscular, a propriocepção e o encurtamento dos tecidos moles; bem como aliviar, assim, as compensaçôes musculoesqueléticas produzidas pela hipertonia e promovendo estabilidade corporal, endireitamento e equilíbrio.

No contexto das crianças com deficiência neuromotora é fundamental favorecer melhor alinhamento biomecânico e estimular movimentos ativos e voluntários para promover a funcionalidade, o alongamento e a consciência e esquema corporal, os quais são favoráveis para melhorar a mobilidade e a postura porque permitem que os movimentos sejam feitos com menor compensação e mais eficiência. Além de trabalhar na perspectiva da plasticidade cerebral, capacidade do sistema nervoso central de modificar suas propriedades morfológicas e funcionais, adaptando-se a alteraçóes internas ou ambientais. A plasticidade neural diz respeito às modificaçóes decorrentes do desenvolvimento, da aprendizagem e das experiências.

Observa-se que o uso de dispositivos como os trajes citados neste estudo tem se difundido na reabilitação, pois atende os princípios das órteses. Contudo, ainda é necessário promover a acessibilidade econômica de tais dispositivos. Assim, a proposta de desenvolvimento da roupa biocinética é bastante pertinente no nosso cenário, pois esta utiliza materiais que respeitam as condiçóes climáticas, bem como reduzem os custos do produto final.

A roupa biocinética ainda está em fase de estudo, porém é possível observar, ao se experimentar a roupa, a melhora da propriocepção, a redução dos reflexos patológicos, restauraçáo dos padróes de movimento e postura apropriados. Novos protótipos estáo sendo desenvolvidos a fim de se melhorar o próprio modelo, facilitando o vestir, bem como confeccionar modelos em caso de comprometimentos em membros superiores.

\section{Referências}

ADLER, S. S.; BECKERS, D.; BUCK, M. PNF Facilitação Neuromuscular Proprioceptiva. 2. ed. São Paulo: Manole, 2007. 
ALVES DE OLIVEIRA, A I. Pesquisa e desenvolvimento infantil. Belém: Conhecimento \& Ciência; 2008.

ASSUMPÇÃO, T. N. Órteses - Princípios Básicos. In: FREITAS, P. P. Reabilitação da Mão. Atheneu: São Paulo, 2005. p. 27.

CARGNin, A. P.; MAZZITELli, C. Proposta de tratamento fisioterapeutico para crianças portadoras de paralisia cerebral espastica, com ênfase nas alteraçôes musculoesqueléticas. Revista Neurociências, São Paulo, v. 11, n. 1, p. 34-39, 2003.

KENDALL, F. P. et al. Músculos: provas e funçôes com postura e dor. 5. ed. Barueri, SP: Manole; 2007.

KONRAD, H. R.; GIRARDI, M.; HELFERT, R. Balance and Aging. Laryngoscope, Saint Louis, v. 109, n. 9, p. 1454-60, Sept 1999. PMid:10499055. http://dx.doi. org/10.1097/00005537-199909000-00019

KOSCIELNY, I. TherasuitTheraSuit - Soft Dynamic Proprioceptive Orthotic. Cerebral Palsy Magazine, Michigan, v. 2, n. 2, p. 8-23, 2004.

LYCRA. Disponível em: <http://www.lycra.com/>. Acesso em: 13 dez. 2012.

MANCINI, M. C. et al. Comparação do desempenho de atividades funcionais em crianças com desenvolvimento normal e crianças com paralisia cerebral. Arquivos de NeuroPsiquiatria, São Paulo, v. 60, n. 2b, p. 446-52, jun. 2002. http://dx.doi.org/10.1590/S0004-282X2002000300020 MOURA, R. C. F. et al. Alteração espirométrica em crianças com mielomeningocele é dependente do nível de lesão funcional. Fisioterapia em Movimento, Curitiba, v. 24, n. 2, p. 231-238, abr./jun. 2011. http://dx.doi. org/10.1590/S0103-51502011000200004

PINHEIRO, J. M. S. Da Iniciação Científica ao TCC: Uma abordagem para os cursos de Tecnologia. Rio de Janeiro: Editora Ciência Moderna Ltda, 2010.

RODRIGUES, A. V. N.; CAVALCANTI, A.; GALVÃO, C. Órtese e Prótese. In: CAVALCANTI, A.; GALVÃO, C. Terapia Ocupacional: fundamentação e prática. Rio de Janeiro: Guanabara Koogan, 2007. p. 435-450.

SILVA, A. C.; ZAMBALDE, A. L. Entre a pesquisa e a inovação tecnológica: o desafio da transformação do conhecimento em desenvolvimento na universidade. In: CONGRESSO DE INICIAÇĀO CIENTÍFICA DA UFLA - CIUFLA, 21., 2008, Lavras. Anais... Lavras: UFLA/PRP, 2008. p. 39.

SPIO. The Official SPIO ${ }^{\mathrm{TM}}$ Site. Disponível em: <www. spioworks. com>. Acesso em: 03 nov. 2012.

TEXSITE. Helanca (fio texturizado). Disponível em: <http://pt.texsite.info/Helanca_(fio_texturizado)>. Acesso em: 15 dez. 2012.

THERATOGS. Rehabmart Newsletter. [on line]. Disponível em: <http://www.rehabmart.com/category/ TheraTogs.htm>. Acesso em: 10 sept. 2012.

THERASUIT METHOD. 2002. Disponível em: <www. suittherapy.com>. Acesso em: 20 jan. 2012.

UBALDO, J. O. P. A utilização do método neuroevolutivo Bobath na paralisia cerebral: intervenção fisioterapêutica domiciliar. 2002.50 f. Trabalho de Conclusão de Curso (Graduação em Fisioterapia)-Universidade do Sul de Santa Catarina, Florianópolis, 2002.

\section{Contribuições dos Autores}

Ana Irene Alves de Oliveira - Estruturação da coleta e análise dos dados, concepção do texto manuscrito, revisão do texto. Larissa Santos Prazeres - Concepção do texto manuscrito, coleta de dados, organização das fontes, revisão do texto.

\section{Notas}

${ }^{1}$ Órtese é um dispositivo acoplado em determinado segmento corporal para modificar as características funcionais do sistema neuromusculoesquelético. Dentre seus objetivos, destacam-se a proteção contra lesões e a prevenção ou correção de deformidades (ASSUMPÇÃO, 2005).

${ }^{2}$ Fio texturizado fabricado originalmente pela empresa Suíça Heberlein \& Co., texturizado a partir de poliamida ou poliéster. O nome é uma marca registada. As características principais desses artigos são a sua elasticidade, suavidade e facilidade novestir (TEXSITE, 2012).

${ }^{3}$ Lycra $^{\circledR}$ é uma marca registrada que identifica uma fibra sintética de grande elasticidade conhecida tecnicamente como elastano ou spandex. Possui elasticidade inigualável e propriedades de recuperação. O fio Lycra ${ }^{\circledR}$ soma conforto, corte, retenção de forma, durabilidade e liberdade de movimento. Isso é obtido graças às exclusivas características do fio, que pode ser esticado até sete vezes seu comprimento inicial e retornar à posição original uma vez liberada a tensão (LYCRA, 2012). 